\title{
Benelux multicentre trial of alpha interferon treatment for chronic hepatitis C: standard $v$ high dose treatment monitored by biochemical and virological markers (interim analysis)
}

\author{
Benelux Multicentre Trial Study Group
}

\begin{abstract}
In an interim analysis of the Benelux trial, no difference in alanine aminotransferase normalisation has been found between the high (6 million units) and standard (3 million units) doses of interferon alfa$2 b$ during the first eight weeks of treatment. The probability of achieving normal alanine aminotransferase activity on at least two successive occasions during treatment is $65 \%$ for the standard dose and $73 \%$ for the higher dose. Measurement of plasma hepatitis $C$ virus-RNA suggests that hepatitis $C$ virus-RNA negativity after four weeks of treatment is a prerequisite for sustained response to interferon alfa-2b.
\end{abstract}

(Gut 1993; supplement: S119-S120)

A standard six month course of treatment with alpha interferon, 3 million units thrice weekly, leads to alanine aminotransferase normalisation in about $50 \%$ of patients with hepatitis $\mathrm{C}$ virus infection. Relapses occur in most of the patients, however, after treatment is stopped. So, conceivably, treatment results might be improved if a higher induction dose is used and if the dosage and duration of treatment are attuned to the effects on alanine aminotransferase and hepatitis $C$ virus-RNA.

\footnotetext{
Methods

Patients were included in the Benelux multicentre trial if they had: (a) history of alanine aminotransferase $\geqslant 2$ times the upper limit of normal, for at least six months; (b) history of exposure to blood products or a positive anti-HCV test; (c) no clinical, histological or serological evidence of other viral, alcoholic, drug, or obesity related, hereditary or autoimmune hepatitis. Patients were randomised to receive either: (a) standard treatment; 3 million units interferon alfa-2b (INTRON A) thrice weekly for 24 weeks; (b) experimental treatment: induction phase of 6 million units interferon alfa-2b thrice weekly for eight weeks, followed by a maintenance phase of titrated doses of interferon 6 to 1 million units thrice weekly until biochemical and virological remission (alanine aminotransferase activity normal, plasma hepatitis C virus-RNA undetectable) was achieved while on 1 million units thrice weekly. Interferon was
}

given by subcutaneous injection. Treatment was withdrawn in cases of non-response after 12 weeks, or after 52 weeks in cases of partial response.

\section{Results}

To date, 244 patients with hepatitis $\mathrm{C}$ virus have been entered into the study. Patient characteristics before treatment are comparable with the standard and experimental treatment group (Table). No statistically significant differences were seen between the groups by either $\chi$ square or Wilcoxon's rank sum test.

Data for 12 weeks are available for 188 patients and 149 patients have been followed for at least six months. No difference in alanine aminotransferase normalisation rate has been found between the high ( 6 million units) and standard (3 million units) induction dose during the first eight weeks, with figures of $53 \%(95 \%$ confidence intervals 46,66$)$ and $54 \%(95 \%$ confidence intervals 44,64$)$, respectively. The probability (actuarial analysis) of reaching normal alanine aminotransferase activity on at least two successive occasions during treatment is $65 \%(95 \%$ confidence intervals 56,74$)$ for the standard scheme and $73 \%$ (95\% confidence intervals 64,82 ) for the experimental scheme, with a plateau after 16 weeks (NS).

The plasma hepatitis $\mathrm{C}$ virus-RNA has been measured in a subgroup of the first consecutive 24 patients from one centre, and studied sequentially to evaluate whether a long term treatment response could be predicted early. Fifteen patients had detectable hepatitis C virus-RNA at four weeks' treatment despite

TABLE Patient characteristics before treatment

\begin{tabular}{lcc}
\hline & \multicolumn{2}{c}{ Interferon treatment schedule } \\
\cline { 2 - 3 } & $\begin{array}{c}\text { Standard } \\
(n=120)\end{array}$ & $\begin{array}{c}\text { Experimental } \\
(n=124)\end{array}$ \\
\hline Men/women & $74 / 46$ & $69 / 55$ \\
Blood exposure & 74 & 79 \\
Anti-HCV positive & 116 & 120 \\
Age & $47 \cdot 1(14)$ & $46 \cdot 7(14)$ \\
Alanine aminotransferase (IU/1) & $168(98)$ & $150(91)$ \\
Hepatitis C virus-RNA positive $\dagger$ & $24 / 27$ & $37 / 39$ \\
Histological examination & & \\
Chronic persistent hepatitis & 20 & 28 \\
Chronic active hepatitis & 65 & 64 \\
Cirrhosis & 21 & 30 \\
\hline
\end{tabular}

${ }^{\star}$ Mean (SD)

†Tested in 66 patients only. 
alanine aminotransferase normalisation in seven. All 15 remained viraemic until the end of treatment (six months), 13 of them with raised transaminase activity. Nine of the 24 patients had undetectable hepatitis $C$ virusRNA at four weeks while seven remained hepatitis $C$ virus-RNA negative until the end of treatment. These results suggest that, when hepatitis $\mathrm{C}$ virus-RNA is detectable after four weeks of interferon treatment, it is predictive for long term non-response. Hepatitis $C$ virusRNA negativity after four weeks of treatment with interferon seems to be a prerequisite for a sustained treatment response. The hepatitis $C$ virus-RNA value at four weeks is of more importance than alanine aminotransferase activity in predicting the long term treatment response $(\mathrm{p}<0 \cdot 001$, Fisher's exact test).

\section{Conclusions}

High dose induction treatment ( $6 v 3$ million units alpha interferon thrice weekly) does not improve the early response rate. Hepatitis C virus-RNA determination at four weeks' treatment seems to be highly predictive for long term treatment outcome.

\section{University Hospital Rotterdam, \\ The Netherlands \\ $\mathrm{J} T$ Brouwer \\ G E M Kleter \\ W C J Hop \\ W G V Quint \\ R A Heijtink \\ S W Schalm}

\section{University Hospital Leuven, Belgium \\ F Nevens \\ J Fevery}

\section{University Hospital Gent, Belgium}

A Elewaut

J Versieck

Erasme University Hospital Brussels, Belgium

M Adler

$\mathrm{N}$ Bourgeois
St Joseph Hospital, Gilly/University Hospital St Luc, Brussels, Belgium

R Brénard

A P Geubel

Academic Medical Center Amsterdam, The Netherlands

R A F M Chamuleau

H W Reesink

P L M Jansen

C Bronkhorst

F J W ten Kate

\section{University Hospital Liège, Belgium}

J Pirotte

\author{
Free University Hospital Brussels, \\ Belgium \\ M L Hautekeete
}

University Hospital Antwerpen, Belgium

P Michielsen

Jolimont Hospital, Haine St Paul, Belgium

J Henrion

St Franciscus Hospital, Rotterdam, The Netherlands

$\mathrm{J}$ W den Ouden

St Anne Hospital, Brussels, Belgium

C de Galoscy

Rijnstaten Hospital, Arnhem, The Netherlands

$\mathrm{R}$ A de Vries

University Hospital Nijmegen, The Netherlands

$\mathrm{J} P$ van Munster

Haven Hospital, Rotterdam, The Netherlands

P J Wismans

Willem Alexander Hospital, s'Hertogenbosch, The Netherlands Th J M van Ditzhuysen 